\title{
ON THE SHAPE OF TETRAHEDRA FROM BISECTION
}

\author{
ANWEI LIU AND BARRY JOE
}

\begin{abstract}
We present a procedure for bisecting a tetrahedron $\mathbf{T}$ successively into an infinite sequence of tetrahedral meshes $\mathscr{T}^{0}, \mathscr{T}^{1}, \mathscr{T}^{2}, \ldots$, which has the following properties: (1) Each mesh $\mathscr{T}^{n}$ is conforming. (2) There are a finite number of classes of similar tetrahedra in all the $\mathscr{T}^{n}, n \geq 0$. (3) For any tetrahedron $\mathbf{T}_{i}^{n}$ in $\mathscr{T}^{n}, \eta\left(\mathbf{T}_{i}^{n}\right) \geq c_{1} \eta(\mathbf{T})$, where $\eta$ is a tetrahedron shape measure and $c_{1}$ is a constant. (4) $\delta\left(\mathbf{T}_{i}^{n}\right) \leq c_{2}(1 / 2)^{n / 3} \delta(\mathbf{T})$, where $\delta\left(\mathbf{T}^{\prime}\right)$ denotes the diameter of tetrahedron $\mathbf{T}^{\prime}$ and $c_{2}$ is a constant.

Estimates of $c_{1}$ and $c_{2}$ are provided. Properties (2) and (3) extend similar results of Stynes and Adler, and of Rosenberg and Stenger, respectively, for the 2-D case. The diameter bound in property (4) is better than one given by Kearfott.
\end{abstract}

\section{INTRODUCTION}

Let $\mathbf{T}\left(t_{0}, t_{1}, t_{2}, t_{3}\right)$ be a tetrahedron with vertices $t_{0}, t_{1}, t_{2}, t_{3}$. Using the midpoint $t$ of one of the edges, $t_{1} t_{2}$ say, and the face $t_{0} t_{3} t$, we can bisect $\mathrm{T}$ into two subtetrahedra $\mathbf{T}_{1}^{1}\left(t_{0}, t_{1}, t, t_{3}\right)$ and $\mathbf{T}_{2}^{1}\left(t_{0}, t, t_{2}, t_{3}\right)$. Next, these two tetrahedra can be bisected, producing four subtetrahedra. This process can be repeated iteratively to produce an infinite sequence of tetrahedral meshes $\mathscr{T}^{0}$, $\mathscr{T}^{1}, \mathscr{T}^{2}, \ldots$, where $\mathscr{T}^{n}$ contains $2^{n}$ tetrahedra.

In the bisection method of [5], which works for simplices of any dimension, the longest edge is always chosen to be bisected. Let $\delta(\mathbf{S})$ denote the diameter (length of longest edge) of a simplex $\mathbf{S}$. In [5] a bound is derived on how fast the diameters of the simplices in the sequence of meshes converge to zero. In the tetrahedron case, this bound is $\delta\left(\mathbf{T}_{i}^{n}\right) \leq(\sqrt{3} / 2)^{\lfloor n / 3\rfloor} \delta(\mathbf{T})$, where $\mathbf{T}_{i}^{n}$ is a tetrahedron in $\mathscr{T}^{n}$. In the two-dimensional or triangle case, $[9,11,1]$ contain results on the bisection method in which the longest edge of each triangle is bisected. In [11] and [1] diameter bounds are given which improve on the bound in [5] (for the 2-D case).

In this paper, we present a bisection procedure for tetrahedra which does not always bisect the longest edge; instead a mapping to a special tetrahedron is

Received by the editor January 19, 1993 and, in revised form, June 28, 1993.

1991 Mathematics Subject Classification. Primary 51M20; Secondary 52B10, 65M50, 65N50.

This work was partially supported by a grant from the Natural Sciences and Engineering Research Council of Canada. 
used to choose the bisected edge. We show that this procedure has the following properties:

(1) Each mesh $\mathscr{T}^{n}$ is conforming, where a conforming mesh is one in which the intersection of any two tetrahedra $\mathbf{T}_{1}, \mathbf{T}_{2}$ of the mesh is either a common face of $\mathbf{T}_{1}$ and $\mathbf{T}_{2}$, or a common edge, or a common vertex, or empty.

(2) There are a finite number of classes of similar tetrahedra in all the $\mathscr{T}^{n}$, $n \geq 0$.

(3) $\eta\left(\mathbf{T}_{i}^{n}\right) \geq c_{1} \eta(\mathbf{T})$, where $\eta$ is a tetrahedron shape measure and $c_{1}$ is a positive constant independent of $\mathbf{T}$.

(4) $\delta\left(\mathbf{T}_{i}^{n}\right) \leq c_{2}(1 / 2)^{n / 3} \delta(\mathbf{T})$, where $c_{2}$ is a positive constant independent of T.

Property (1) is not generally satisfied if the longest edge is always bisected. Property (2) generalizes a similar result for the 2-D case, given in [11] and [1]. The diameter bound in property (4) is better than that given in [5]. Property (3) is important for the local refinement of tetrahedral finite element meshes in which it is desired that poorly-shaped tetrahedra be avoided [3] (to get better approximations and to avoid ill-conditioned matrices in the finite element method). In two dimensions, it is shown in [9] that $\theta \geq \alpha / 2$, where $\alpha$ is the minimum interior angle in the original triangle and $\theta$ is any interior angle in a refined triangle. So property (3) extends this result to three dimensions (using a different shape measure and a smaller constant). There has been no previous result on the shape of the refined tetrahedra.

These properties should be useful in designing local refinement algorithms for tetrahedral meshes, which are a generalization of Rivara's algorithms for triangular meshes $[6,7,8]$. After describing the new tetrahedron shape measure $\eta$ in $\S 2$ and our bisection procedure based on a special tetrahedron in $\S 3$, we establish the above properties in $\S 4$. Estimates of the constants $c_{1}$ and $c_{2}$ are provided in $\S 5$.

\section{A NEW TETRAHEDRON SHAPE MEASURE}

Tetrahedron shape measures are used to measure the shape of different tetrahedra. Two commonly used shape measures are the aspect ratio $\rho$ (ratio of inradius to circumradius) and the minimum solid angle $\theta_{\min }$ (each tetrahedron has 4 solid angles) [3, 4]. For these two measures, the highest value occurs for a regular tetrahedron and values approaching zero occur for poorly-shaped tetrahedra (e.g., tetrahedra with four nearly coplanar vertices). The expressions for both $\rho$ and $\theta_{\min }$ are complicated. In this section, we introduce a new shape measure $\eta$ with a simple expression, and use it to prove properties (3) and (4) of our bisection procedure. More details about the application of $\eta$ in local refinement algorithms and some comparisons between $\eta, \rho$, and $\theta_{\min }$ will be addressed in a later paper. 


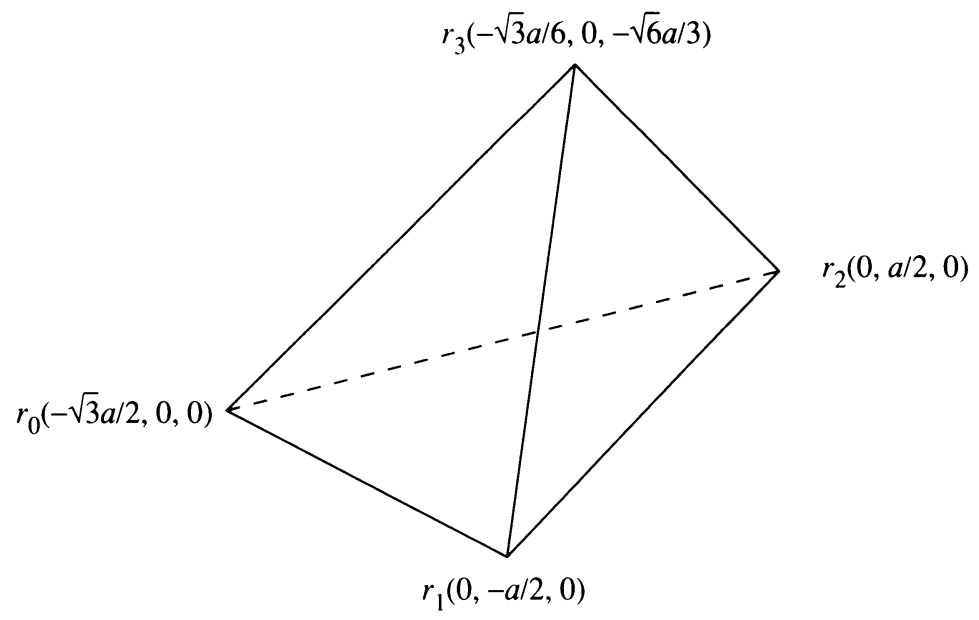

FigURE 1. Regular tetrahedron $\mathbf{R}$

Definition 1. For any (nondegenerate) tetrahedron $\mathbf{T}\left(t_{0}, t_{1}, t_{2}, t_{3}\right)$, define the 3 by 3 nonsingular matrix $T=\left[t_{1}-t_{0}, t_{2}-t_{0}, t_{3}-t_{0}\right]$. Note that the matrix has the same name as the tetrahedron but italic font is used instead of bold font, and $T$ depends on the ordering of vertices of $T$. For any two tetrahedra $\mathbf{S}\left(s_{0}, s_{1}, s_{2}, s_{3}\right)$ and $\mathbf{T}\left(t_{0}, t_{1}, t_{2}, t_{3}\right)$, define the 3 by 3 matrices $M(\mathbf{S}, \mathbf{T})=$ $T S^{-1}$ and $A(\mathbf{S}, \mathbf{T})=M^{T}(\mathbf{S}, \mathbf{T}) M(\mathbf{S}, \mathbf{T})$. Note that $M$ and $A$ also depend on the ordering of tetrahedron vertices, and $M$ is the matrix involved in the affine transformation from points of $\mathbf{S}$ to points of $\mathbf{T}$ such that $t_{i}=M(\mathbf{S}, \mathbf{T}) s_{i}+b$, $0 \leq i \leq 3$, where $b=t_{0}-M(\mathbf{S}, \mathbf{T}) s_{0}$.

Definition 2. Let $\mathbf{T}\left(t_{0}, t_{1}, t_{2}, t_{3}\right)$ be any tetrahedron, and $\mathbf{R}\left(r_{0}, r_{1}, r_{2}, r_{3}\right)$ ke a regular tetrahedron with the same volume as $\mathbf{T}$, e.g., as shown in Figure 1. Define the tetrahedron shape measure $\eta(\mathbf{T})=3 \sqrt[3]{\lambda_{1} \lambda_{2} \lambda_{3}} /\left(\lambda_{1}+\lambda_{2}+\lambda_{3}\right)$, where $\lambda_{1}, \lambda_{2}$, and $\lambda_{3}$ are the eigenvalues of the matrix $A(\mathbf{R}, \mathbf{T})$. Note that the three eigenvalues are positive since $A$ is positive definite, and $0<\eta(\mathbf{T}) \leq 1$ with $\eta(\mathbf{T})=1$ if and only if $\lambda_{1}=\lambda_{2}=\lambda_{3}$.

Theorem 1. For any tetrahedron $\mathbf{T}\left(t_{0}, t_{1}, t_{2}, t_{3}\right)$, we have

$$
\eta(\mathbf{T})=\frac{12(3 v)^{2 / 3}}{\sum_{i=1}^{6} l_{i}^{2}}
$$

where $v$ is the volume of $\mathbf{T}$ and the $l_{i}$ are the lengths of the edges of $\mathbf{T}$. Furthermore, $\eta(\mathbf{T})$ is independent of the ordering of vertices of $\mathbf{T}, \mathbf{R}$ and of the vertex coordinates of $\mathbf{R}$.

Proof. We first let $\mathbf{R}\left(r_{0}, r_{1}, r_{2}, r_{3}\right)$ be the regular tetrahedron with the same volume as $\mathbf{T}\left(t_{0}, t_{1}, t_{2}, t_{3}\right)$ and the vertex coordinates of Figure 1 . Let $T=$ 
$\left[t_{1}-t_{0}, t_{2}-t_{0}, t_{3}-t_{0}\right]$ and $R=\left[r_{1}-r_{0}, r_{2}-r_{0}, r_{3}-r_{0}\right]$. Then

$$
R=a\left[\begin{array}{ccc}
\sqrt{3} / 2 & \sqrt{3} / 2 & \sqrt{3} / 3 \\
-1 / 2 & 1 / 2 & 0 \\
0 & 0 & \sqrt{6} / 3
\end{array}\right], \quad R^{-1}=\frac{1}{a}\left[\begin{array}{ccc}
1 / \sqrt{3} & -1 & -1 / \sqrt{6} \\
1 / \sqrt{3} & 1 & -1 / \sqrt{6} \\
0 & 0 & 3 / \sqrt{6}
\end{array}\right]
$$

where $a=(6 \sqrt{2} v)^{1 / 3}$ and $v$ is the volume of $\mathbf{T}$. Let $d_{i j}=\left(t_{j}-t_{i}\right)^{T}\left(t_{j}-t_{i}\right)$, $0 \leq i<j \leq 3$. Then

(2) $T^{T} T=\left[\begin{array}{ccc}d_{01} & \left(d_{01}+d_{02}-d_{12}\right) / 2 & \left(d_{01}+d_{03}-d_{13}\right) / 2 \\ \left(d_{01}+d_{02}-d_{12}\right) / 2 & d_{02} & \left(d_{02}+d_{03}-d_{23}\right) / 2 \\ \left(d_{01}+d_{03}-d_{13}\right) / 2 & \left(d_{02}+d_{03}-d_{23}\right) / 2 & d_{03}\end{array}\right]$.

From (1), (2), and $A(\mathbf{R}, \mathbf{T})=M^{T}(\mathbf{R}, \mathbf{T}) M(\mathbf{R}, \mathbf{T})=\left(R^{-1}\right)^{T} T^{T} T R^{-1}$, we obtain

(3) $A(\mathbf{R}, \mathbf{T})=\frac{1}{a^{2}}\left[\begin{array}{ccc}\left(2 d_{01}+2 d_{02}-d_{12}\right) / 3 & \# & \# \\ \# & d_{12} & \# \\ \# & \# & \left(3 d_{03}+3 d_{13}+3 d_{23}-d_{01}-d_{02}-d_{12}\right) / 6\end{array}\right]$,

where \# denotes a value which is irrelevant. Then

$$
\begin{aligned}
\lambda_{1}+\lambda_{2}+\lambda_{3} & =\operatorname{trace}(A(\mathbf{R}, \mathbf{T})) \\
& =\left(d_{01}+d_{02}+d_{03}+d_{12}+d_{13}+d_{23}\right) /\left(2 a^{2}\right) .
\end{aligned}
$$

Since $\mathbf{R}$ and $\mathbf{T}$ have the same volume, $\operatorname{det}(M(\mathbf{R}, \mathbf{T}))= \pm 1$. So

$$
\lambda_{1} \lambda_{2} \lambda_{3}=\operatorname{det}(A(\mathbf{R}, \mathbf{T}))=1 .
$$

From (4), (5), and Definition 2,

$$
\eta(\mathbf{T})=\frac{3 \sqrt[3]{\operatorname{det}(A(\mathbf{R}, \mathbf{T}))}}{\operatorname{trace}(A(\mathbf{R}, \mathbf{T}))}=\frac{12(3 v)^{2 / 3}}{\sum_{i=1}^{6} l_{i}^{2}}
$$

where the $l_{i}$ are the lengths of the edges of $\mathbf{T}$.

Now we allow the vertices of $\mathbf{T}$ and $\mathbf{R}$ to be permuted and different vertex coordinates for $\mathbf{R}$. Let $\bar{T}, \bar{R}, \bar{M}(\mathbf{R}, \mathbf{T})$, and $\bar{A}(\mathbf{R}, \mathbf{T})$ be the resulting matrices. Then $\bar{T}=T P_{1} L_{1} P_{2}$ and $\bar{R}=Q R P_{3} L_{2} P_{4}$, where $Q$ is an orthogonal matrix, the $P_{i}$ are permutation matrices, and each $L_{i}$ is either the identity matrix $I$ or

$$
L=\left[\begin{array}{rrr}
1 & 0 & 0 \\
0 & 1 & 0 \\
-1 & -1 & -1
\end{array}\right]
$$

because $\left[t_{1}-t_{3}, t_{2}-t_{3}, t_{0}-t_{3}\right]=\left[t_{1}-t_{0}, t_{2}-t_{0}, t_{3}-t_{0}\right] L=T L$. Since $L^{-1}=L$, we have

$$
\begin{aligned}
\bar{A}(\mathbf{R}, \mathbf{T}) & =\left(\bar{R}^{-1}\right)^{T} \bar{T}^{T} \bar{T} \bar{R}^{-1} \\
& =Q\left(R^{-1}\right)^{T} P_{3} L_{2}^{T} P_{4} P_{2}^{T} L_{1}^{T} P_{1}^{T} T^{T} T P_{1} L_{1} P_{2} P_{4}^{T} L_{2} P_{3}^{T} R^{-1} Q^{T}
\end{aligned}
$$


Let $\hat{A}(\mathbf{R}, \mathbf{T})=Q^{T} \bar{A}(\mathbf{R}, \mathbf{T}) Q$. If $P$ is a permutation matrix, then $P^{T} T^{T} T P$ just applies a symmetric permutation to the matrix of (2). Similarly,

$$
L^{T} T^{T} T L=\left[\begin{array}{ccc}
d_{13} & \left(d_{13}+d_{23}-d_{12}\right) / 2 & \left(d_{03}+d_{13}-d_{01}\right) / 2 \\
\left(d_{13}+d_{23}-d_{12}\right) / 2 & d_{23} & \left(d_{03}+d_{23}-d_{02}\right) / 2 \\
\left(d_{03}+d_{13}-d_{01}\right) / 2 & \left(d_{03}+d_{23}-d_{02}\right) / 2 & d_{03}
\end{array}\right]
$$

Therefore, $\hat{A}(\mathbf{R}, \mathbf{T})$ has the same form as (3), with the $d_{i j}$ permuted, and (4), (5), and (6) are unchanged if $A$ is replaced with $\hat{A}$. Finally, the eigenvalues $\lambda_{i}$ of $\hat{A}(\mathbf{R}, \mathbf{T})$ and $\bar{A}(\mathbf{R}, \mathbf{T})=Q \hat{A}(\mathbf{R}, \mathbf{T}) Q^{T}$ are identical, so $\eta(\mathbf{T})=$ $12(3 v)^{2 / 3} / \sum_{i=1}^{6} l_{i}^{2}$ is independent of the ordering of vertices of $\mathbf{T}, \mathbf{R}$ and of the vertex coordinates of $\mathbf{R}$.

Now we give a geometric explanation of $\eta(\mathbf{T})$. Let $O$ be the inscribed sphere in the regular tetrahedron $\mathbf{R}$ and $r$ be its radius. The affine transformation $y=M(\mathbf{R}, \mathbf{T}) x+b$, which transforms the points of $\mathbf{R}$ into the points $\mathbf{T}$, transforms the sphere $O$ into an inscribed ellipsoid $E$ in $\mathbf{T}$. Let the equation of $O$ be

$$
\left(x+b_{0}\right)^{T}\left(x+b_{0}\right)=r^{2} .
$$

Then the equation of $E$ is

$$
\left(y+b_{1}\right)^{T}\left(M^{-1}(\mathbf{R}, \mathbf{T})\right)^{T} M^{-1}(\mathbf{R}, \mathbf{T})\left(y+b_{1}\right)=r^{2} .
$$

Let $\alpha, \beta, \gamma$ be the half-lengths of the three principal axes inside the ellipsoid, and $\lambda_{1}, \lambda_{2}$, and $\lambda_{3}$ be the eigenvalues of $M^{T}(\mathbf{R}, \mathbf{T}) M(\mathbf{R}, \mathbf{T})$. After a translation and a rotation, the equation of the ellipsoid becomes

$$
\frac{x_{1}^{2}}{\lambda_{1}}+\frac{x_{2}^{2}}{\lambda_{2}}+\frac{x_{3}^{2}}{\lambda_{3}}=r^{2}
$$

where $\left(x_{1}, x_{2}, x_{3}\right)$ is any point on the ellipsoid. So $\alpha^{2}=\lambda_{1} r^{2}, \beta^{2}=\lambda_{2} r^{2}$, and $\gamma^{2}=\lambda_{3} r^{2}$. Since $\lambda_{1} \lambda_{2} \lambda_{3}=1$, we have $r^{2}=\sqrt[3]{\alpha^{2} \beta^{2} \gamma^{2}}$. From Definition 2,

$$
\eta(\mathbf{T})=\frac{3 \sqrt[3]{\lambda_{1} \lambda_{2} \lambda_{3}}}{\lambda_{1}+\lambda_{2}+\lambda_{3}}=\frac{3 \sqrt[3]{\alpha^{2} \beta^{2} \gamma^{2}}}{\alpha^{2}+\beta^{2}+\gamma^{2}}
$$

So $\eta(\mathbf{T})$ is the ratio of the geometric mean to the arithmetic mean of $\alpha^{2}, \beta^{2}$, and $\gamma^{2}$. In some sense we can say that $\eta(\mathbf{T})$ reflects the shape of the inscribed ellipsoid $E$ and hence the shape of $\mathbf{T}$.

From Definition 2, Theorem 1, and the above explanation, it follows that $\eta(\mathbf{T})=1$ if and only if $\mathbf{T}$ is a regular tetrahedron, and $\eta(\mathbf{T})$ approaches zero for poorly-shaped tetrahedra.

\section{BISECTION PROCEDURE BASED ON A SPECIAL TETRAHEDRON}

When a tetrahedron is bisected, the two resulting subtetrahedra are generally not similar to each other or the original tetrahedron. So we try to design a bisection procedure that creates a finite number of classes of similar tetrahedra. To this end, we need the special tetrahedron $\mathbf{P}$ shown in Figure 3. Let $\left|q_{i} q_{j}\right|$ 


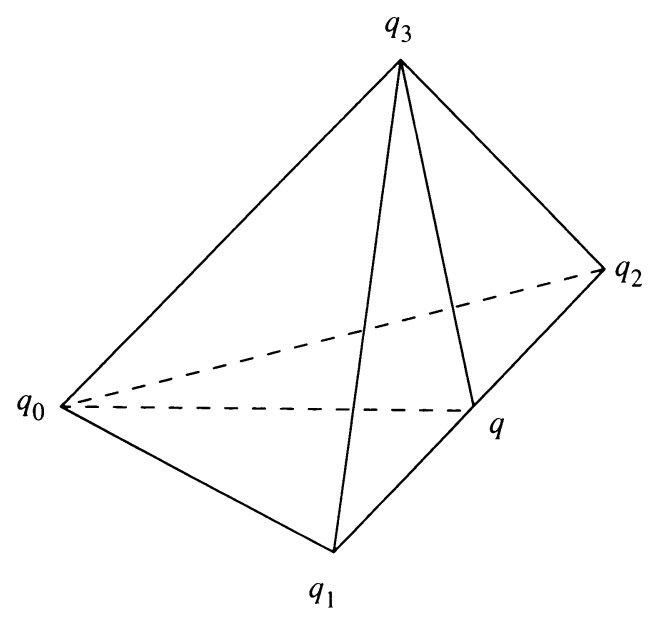

Figure 2. Tetrahedron $\mathbf{Q}$ for Lemmas 1 and 2

denote the length of edge $q_{i} q_{j}$. The following two lemmas, which are proved in [10], are needed for Theorem 2 below.

Lemma 1. Let $\mathbf{Q}\left(q_{0}, q_{1}, q_{2}, q_{3}\right)$ be a tetrahedron with $\left|q_{0} q_{1}\right|=\left|q_{0} q_{2}\right|$ and $\left|q_{1} q_{3}\right|=\left|q_{2} q_{3}\right|$, and let $q$ be the midpoint of $q_{1} q_{2}$ (see Figure 2). Then $\mathbf{Q}_{1}\left(q_{0}, q_{1}, q, q_{3}\right)$ is similar to $\mathbf{Q}_{2}\left(q_{0}, q, q_{2}, q_{3}\right)$.

Lemma 2. Let $\mathbf{Q}\left(q_{0}, q_{1}, q_{2}, q_{3}\right)$ be a tetrahedron with $\left|q_{0} q_{1}\right|=\left|q_{2} q_{3}\right|$ and $\left|q_{0} q_{2}\right|=\left|q_{1} q_{3}\right|$, and let $q$ be the midpoint of $q_{1} q_{2}$ (see Figure 2). Then $\mathbf{Q}_{1}\left(q_{0}, q_{1}, q, q_{3}\right)$ is similar to $\mathbf{Q}_{2}\left(q_{0}, q, q_{2}, q_{3}\right)$.

Theorem 2. In the first three levels of longest edge bisection applied to the special tetrahedron $\mathbf{P}$, the subtetrahedra at the same level are similar to each other, and the subtetrahedra at the third level are all similar to $\mathbf{P}$ (see Figure 3 ).

Proof. Let $p_{i j}=\left(p_{i}+p_{j}\right) / 2, i<j$. The longest edge of $\mathbf{P}\left(p_{0}, p_{1}, p_{2}, p_{3}\right)$ is $p_{1} p_{2}$ with $\left|p_{1} p_{2}\right|=2 a$. First, $\mathbf{P}$ is bisected into two subtetrahedra $\mathbf{P}_{1}^{1}\left(p_{0}, p_{1}, p_{3}, p_{12}\right)$ and $\mathbf{P}_{2}^{1}\left(p_{0}, p_{2}, p_{3}, p_{12}\right)$. Sirce $\left|p_{0} p_{1}\right|=\left|p_{0} p_{2}\right|=\sqrt{6} a / 2$ and $\left|p_{1} p_{3}\right|=\left|p_{2} p_{3}\right|=\sqrt{2} a$, these two subtetrahedra are similar to each other by Lemma 1 . Next, we only need to consider the subtetrahedron $\mathbf{P}_{1}^{1}\left(p_{0}, p_{1}, p_{3}, p_{12}\right)$, whose longest edge is $p_{1} p_{3}$ with $\left|p_{1} p_{3}\right|=\sqrt{2} a$. It is bisected into two subtetrahedra $\mathbf{P}_{1}^{2}\left(p_{0}, p_{1}, p_{12}, p_{13}\right)$ and $\mathbf{P}_{2}^{2}\left(p_{0}, p_{3}, p_{12}, p_{13}\right)$. Since $\left|p_{0} p_{1}\right|=\left|p_{0} p_{3}\right|=$ $\sqrt{6} a / 2$ and $\left|p_{1} p_{12}\right|=\left|p_{3} p_{12}\right|=a$, these two subtetrahedra are similar to each other by Lemma 1 . Finally, we only need to consider the subtetrahedron $\mathbf{P}_{1}^{2}\left(p_{0}, p_{1}, p_{12}, p_{13}\right)$, whose longest edge is $p_{0} p_{1}$ with $\left|p_{0} p_{1}\right|=\sqrt{6} a / 2$. It is bisected into two subtetrahedra $\mathbf{P}_{1}^{3}\left(p_{0}, p_{12}, p_{13}, p_{01}\right)$ and $\mathbf{P}_{2}^{3}\left(p_{1}, p_{12}, p_{13}, p_{01}\right)$. Since $\left|p_{0} p_{12}\right|=\left|p_{1} p_{13}\right|=a / \sqrt{2}$ and $\left|p_{0} p_{13}\right|=\left|p_{1} p_{12}\right|=a$, these two subtetrahedra are similar to each other by Lemma 2.

Since $\mathbf{P}_{2}^{3}\left(p_{1}, p_{12}, p_{13}, p_{01}\right)$ is similar to $\mathbf{P}$, it follows that after three levels of bisections, the eight subtetrahedra $\mathbf{P}_{1}^{3}, \mathbf{P}_{2}^{3}, \mathbf{P}_{3}^{3}\left(p_{0}, p_{12}, p_{13}, p_{03}\right)$, 


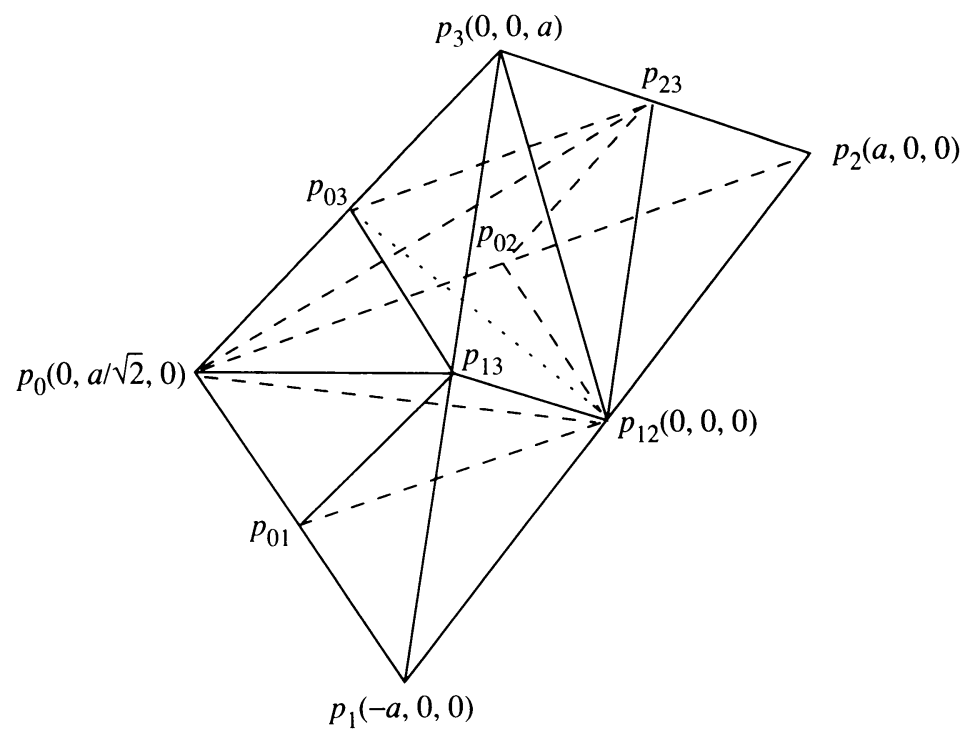

FIGURE 3. Special tetrahedron $\mathbf{P}$; other coordinates are $p_{01}(-a / 2, \sqrt{2} a / 4,0), p_{02}(a / 2, \sqrt{2} a / 4,0), p_{03}(0, \sqrt{2} a / 4, a / 2)$, $p_{13}(-a / 2,0, a / 2), p_{23}(a / 2,0, a / 2)$

$\mathbf{P}_{4}^{3}\left(p_{3}, p_{12}, p_{13}, p_{03}\right), \mathbf{P}_{5}^{3}\left(p_{0}, p_{12}, p_{23}, p_{02}\right), \mathbf{P}_{6}^{3}\left(p_{2}, p_{12}, p_{23}, p_{02}\right), \mathbf{P}_{7}^{3}\left(p_{0}, p_{12}\right.$, $\left.p_{23}, p_{03}\right), \quad \mathbf{P}_{8}^{3}\left(p_{3}, p_{12}, p_{23}, p_{03}\right)$ are all similar to the original tetrahedron P.

It follows from Theorem 2 that if $\mathbf{P}$ is iteratively bisected by the longest edge to an arbitrary number of levels, any subtetrahedron at level $3 k, 3 k+1$, or $3 k+2$ is similar to $\mathbf{P}\left(p_{0}, p_{1}, p_{2}, p_{3}\right), \mathbf{P}_{1}^{1}\left(p_{0}, p_{1}, p_{3}, p_{12}\right)$, or $\mathbf{P}_{1}^{2}\left(p_{0}, p_{1}, p_{12}, p_{13}\right)$, respectively, for $k=0,1, \ldots$ Hence, we define a subtetrahedron at level $3 k$, $3 k+1$, or $3 k+2$ to be a tetrahedron of type $\mathbf{P}^{0}, \mathbf{P}^{1}$, or $\mathbf{P}^{2}$, respectively.

We now present a bisection procedure for iteratively bisecting any tetrahedron $\mathbf{T}$ to $n$ levels. Let $\mathbf{P}$ be the special tetrahedron of Figure 3 such that $\mathbf{T}$ and $\mathbf{P}$ have the same volume.

(a) Transform $\mathbf{T}$ to $\mathbf{P}$ by the affine transformation $y=M^{-1}(\mathbf{P}, \mathbf{T}) x+b_{0}$.

(b) Iteratively bisect $\mathbf{P}$ to $n$ levels by always bisecting the longest edge.

(c) Transform all subtetrahedra $\mathbf{P}_{i}^{n}$ of $\mathbf{P}$ back to subtetrahedra $\mathbf{T}_{i}^{n}$ of $\mathbf{T}$ using the inverse affine transformation $y=M(\mathbf{P}, \mathbf{T}) x+b_{1}$.

Note that in the subtetrahedra of $\mathbf{T}$, the longest edge may not be the one bisected.

\section{Properties of Bisection pRocedure}

In this section, we prove the four properties of the bisection procedure stated in $\S 1$. Let $\mathscr{T}^{n}$ be the mesh of $2^{n}$ subtetrahedra of $\mathbf{T}$ produced by $n$ levels of bisection. Let $\mathbf{R}$ be the regular tetrahedron of Figure 1 such that $\mathbf{T}, \mathbf{P}$, and $\mathbf{R}$ have the same volume. 
Theorem 3. The mesh $\mathscr{T}^{n}$ is conforming.

Proof. In view of the affine transformation used in the bisection procedure, it suffices to prove that the mesh $\mathscr{P}^{n}$ of subtetrahedra in $\mathbf{P}$ is conforming. By considering the first three levels of bisection of $\mathbf{P}$, it is easily seen that at any level, each subtetrahedron of $\mathbf{P}$ has only one longest edge and all of the longest edges of the subtetrahedra have the same length (e.g., after the first level, the two subtetrahedra have longest edges $p_{1} p_{3}$ and $p_{2} p_{3}$, respectively, and $\left|p_{1} p_{3}\right|=\left|p_{2} p_{3}\right|=\sqrt{2} a$, etc.). So the midpoint of any longest edge $e$ is also a bisecting point of any other subtetrahedra incident on $e$. Hence $\mathscr{P}^{n}$ is a conforming mesh.

In the above proof, we have assumed that each subtetrahedron is bisected to the same level. This is not necessary in order to get a conforming mesh. For example, after $\mathbf{P}$ is bisected, if only $\mathbf{P}_{1}^{1}\left(p_{0}, p_{1}, p_{3}, p_{12}\right)$ is bisected at the first level, and the same for $\mathbf{P}_{1}^{2}\left(p_{0}, p_{1}, p_{12}, p_{13}\right)$ at the second level, then the resulting mesh is still conforming. This property can be used to smoothly extend local refinements to adjacent tetrahedra. For the case when the bisection starts with more than one tetrahedron, it is not easy to guarantee the conformity of the resulting mesh by using the above procedure alone. In a later paper, we will present local refinement algorithms which use this procedure and others to guarantee conformity.

Theorem 4. There are a finite number of classes of similar tetrahedra in all the $\mathscr{T}^{n}, n \geq 0$.

Proof. We define two tetrahedra to be in the same equivalence class if one can be transformed into the other by translation and uniform scaling (i.e., the scale factors for the three coordinate axes are the same). So any two tetrahedra in the same equivalence class are similar to each other after any affine transformation. In order to prove the theorem, it suffices to prove that all subtetrahedra $\mathbf{P}_{i}^{n}$, $n \geq 0$, generated by the bisection procedure are only in a finite number of equivalence classes. First we prove that the tetrahedra of type $\mathbf{P}^{0}$ are only in a finite number of equivalence classes.

After three levels of bisection, by Theorem 2, all eight subtetrahedra $\mathbf{P}_{i}^{3}$ are similar to $\mathbf{P}$. Let these tetrahedra be labeled $\mathbf{P}_{1}^{3}\left(p_{01}, p_{1}, p_{12}, p_{13}\right)$, $\mathbf{P}_{2}^{3}\left(p_{02}, p_{12}, p_{2}, p_{23}\right), \mathbf{P}_{3}^{3}\left(p_{01}, p_{0}, p_{13}, p_{12}\right), \mathbf{P}_{4}^{3}\left(p_{03}, p_{13}, p_{0}, p_{12}\right), \mathbf{P}_{5}^{3}\left(p_{03}, p_{3}, p_{12}, p_{13}\right)$, $\mathbf{P}_{6}^{3}\left(p_{02}, p_{0}, p_{23}, p_{12}\right), \mathbf{P}_{7}^{3}\left(p_{03}, p_{3}, p_{12}, p_{23}\right)$, and $\mathbf{P}_{8}^{3}\left(p_{03}, p_{23}, p_{0}, p_{12}\right)$. Let $M_{i}$ $=M\left(\mathbf{P}, \mathbf{P}_{i}^{3}\right), 1 \leq i \leq 8$. From the coordinates of Figure 3, we obtain $M_{1}=M_{2}=1 / 2 I$, where $I$ is the identity matrix, and

$$
M_{3}=\frac{1}{4}\left[\begin{array}{ccc}
-1 & -\sqrt{2} & 1 \\
-\sqrt{2} & 0 & -\sqrt{2} \\
1 & -\sqrt{2} & -1
\end{array}\right], \quad M_{4}=\frac{1}{4}\left[\begin{array}{ccc}
1 & \sqrt{2} & 1 \\
\sqrt{2} & 0 & -\sqrt{2} \\
-1 & \sqrt{2} & -1
\end{array}\right],
$$




$$
\begin{gathered}
M_{5}=\frac{1}{2}\left[\begin{array}{ccc}
0 & 0 & -1 \\
0 & 1 & 0 \\
-1 & 0 & 0
\end{array}\right], M_{6}=\frac{1}{4}\left[\begin{array}{ccc}
1 & \sqrt{2} & -1 \\
-\sqrt{2} & 0 & -\sqrt{2} \\
1 & -\sqrt{2} & -1
\end{array}\right], \\
M_{7}=\frac{1}{2}\left[\begin{array}{ccc}
0 & 0 & 1 \\
0 & 1 & 0 \\
-1 & 0 & 0
\end{array}\right], \quad M_{8}=\frac{1}{4}\left[\begin{array}{ccc}
-1 & -\sqrt{2} & -1 \\
\sqrt{2} & 0 & -\sqrt{2} \\
-1 & \sqrt{2} & -1
\end{array}\right] .
\end{gathered}
$$

Note that the vertices of the $\mathbf{P}_{i}^{3}$ are ordered so that $M_{i}=1 / 2 Q_{i}$, where $Q_{i}$ is an orthogonal matrix.

Let

$$
\operatorname{diag}\left(a_{1}, a_{2}, a_{3}\right)=\left[\begin{array}{ccc}
a_{1} & 0 & 0 \\
0 & a_{2} & 0 \\
0 & 0 & a_{3}
\end{array}\right], \quad \operatorname{skew}\left(a_{1}, a_{2}, a_{3}\right)=\left[\begin{array}{ccc}
0 & 0 & a_{1} \\
0 & a_{2} & 0 \\
a_{3} & 0 & 0
\end{array}\right]
$$

The following equations can be obtained by straightforward computation:

$$
\begin{aligned}
& M_{6}=\operatorname{diag}(-1,1,1) M_{3}, M_{8}=\operatorname{diag}(-1,1,1) M_{4}, M_{3}^{2}=1 / 4 I, \\
& M_{3} M_{4}=1 / 4 \operatorname{diag}(-1,-1,1), M_{4} M_{3}=1 / 2 \operatorname{diag}(-1,-1,1) M_{4}, \\
& M_{3} M_{5}=1 / 2 \operatorname{diag}(1,-1,1) M_{3}, M_{5} M_{3}=1 / 2 \operatorname{skew}(-1,1,-1) M_{3}, \\
& M_{3} M_{6}=1 / 2 \operatorname{skew}(-1,-1,1) M_{4}, M_{6} M_{3}=1 / 4 \operatorname{diag}(-1,1,1), \\
& M_{3} M_{7}=1 / 2 \operatorname{diag}(-1,1,-1) M_{4}, M_{7} M_{3}=1 / 2 \operatorname{skew}(1,1,-1) M_{3}, \\
& M_{3} M_{8}=1 / 2 \operatorname{skew}(-1,-1,1) M_{3}, M_{8} M_{3}=1 / 2 \operatorname{diag}(1,-1,1) M_{4}, \\
& M_{4}^{2}=1 / 2 \operatorname{diag}(-1,-1,1) M_{3}, M_{4} M_{5}=1 / 2 \operatorname{skew}(1,1,1) M_{4}, \\
& M_{5} M_{4}=1 / 2 \operatorname{skew}(-1,1,-1) M_{4}, M_{4} M_{6}=1 / 4 \operatorname{skew}(-1,1,-1), \\
& M_{6} M_{4}=1 / 4 \operatorname{diag}(1,-1,1), M_{4} M_{7}=1 / 2 \operatorname{skew}(-1,-1,-1) M_{3}, \\
& M_{7} M_{4}=1 / 2 \operatorname{skew}(1,1,-1) M_{4}, M_{4} M_{8}=1 / 4 \operatorname{skew}(-1,-1,1), \\
& M_{8} M_{4}=1 / 2 \operatorname{diag}(1,-1,1) M_{3}, M_{5}^{2}=1 / 4 I, \\
& M_{5} M_{6}=1 / 2 \operatorname{skew}(-1,1,1) M_{3}, M_{6} M_{5}=1 / 2 \operatorname{diag}(-1,-1,1) M_{3}, \\
& M_{5} M_{7}=1 / 4 \operatorname{diag}(1,1,-1), M_{7} M_{5}=1 / 4 \operatorname{diag}(-1,1,1), \\
& M_{5} M_{8}=1 / 2 \operatorname{skew}(-1,1,1) M_{4}, M_{8} M_{5}=1 / 2 \operatorname{skew}(-1,1,1) M_{4}, \\
& M_{6}^{2}=1 / 2 \operatorname{skew}(1,-1,1) M_{4}, M_{6} M_{7}=1 / 2 \operatorname{diag}(1,1,-1) M_{4}, \\
& M_{7} M_{6}=1 / 2 \operatorname{skew}(1,1,1) M_{3}, M_{6} M_{8}=1 / 2 \operatorname{skew}(1,-1,1) M_{3}, \\
& M_{8} M_{6}=1 / 4 \operatorname{skew}(1,1,-1), M_{7}^{2}=1 / 4 \operatorname{diag}(-1,1,-1), \\
& M_{7} M_{8}=1 / 2 \operatorname{skew}(1,1,1) M_{4}, M_{8} M_{7}=1 / 2 \operatorname{skew}(1,-1,-1) M_{3}, \\
& M_{8}^{2}=1 / 4 \operatorname{skew}(1,-1,1) .
\end{aligned}
$$

After six levels of bisection, with a suitable ordering of vertices of $\mathbf{P}_{i}^{6}$, we get $M\left(\mathbf{P}, \mathbf{P}_{i}^{6}\right)=M\left(\mathbf{P}_{j}^{3}, \mathbf{P}_{i}^{6}\right) M\left(\mathbf{P}, \mathbf{P}_{j}^{3}\right)$, where $\mathbf{P}_{i}^{6}$ is a subtetrahedron of $\mathbf{P}_{j}^{3}$, so

$$
M\left(\mathbf{P}, \mathbf{P}_{i}^{6}\right)=P_{i}^{6}\left(P_{j}^{3}\right)^{-1} M_{j}=M_{j} P_{l}^{3}\left(M_{j} P\right)^{-1} M_{j}=M_{j} P_{l}^{3} P^{-1}=M_{j} M_{l}
$$

for some $l$. By induction, after $3 k$ levels of bisection, for each subtetrahedron $\mathbf{P}_{i}^{3 k}$ with a suitable ordering of vertices $p_{i j}^{3 k}$, we have

$$
p_{i j}^{3 k}=M_{l 1} M_{l 2} \cdots M_{l k} p_{j}+b_{i}^{3 k}, \quad 0 \leq j \leq 3,
$$


where each $M_{l m}, 1 \leq m \leq k$, is one of the $M_{i}, 1 \leq i \leq 8$. Let $\mathscr{S}$ be the set of all diagonal and skew diagonal matrices with elements 1 or -1 . Obviously, $\mathscr{S}$ is closed under matrix multiplication, and $|\mathscr{S}|=2^{3}+2^{3}=16$. So, by the above equations, $M_{l 1} M_{l 2} \cdots M_{l k}=f D M$, where $f$ is a scale factor, $D$ is an element of $\mathscr{S}$, and $M$ is either $I, M_{3}$, or $M_{4}$. Note that $D$ and $-D$ can be considered to be the same matrix of $\mathscr{S}$ because of the factor $f$. Therefore, the number of different equivalence classes of tetrahedra of type $\mathbf{P}^{0}$ in $\mathscr{P}{ }^{n}$, $n \geq 0$, is $\leq 3 \times 8=24$. Note that a type $P^{1}$ tetrahedron is generated by bisecting the longest edge of a type $\mathbf{P}^{0}$ tetrahedron. Since the longest edge of a tetrahedron is still the longest edge under translation and uniform scaling, each one of the 24 possible equivalence classes of tetrahedra of type $\mathbf{P}^{0}$ creates two equivalence classes of tetrahedra of type $\mathbf{P}^{1}$. So the number of different equivalence classes of tetrahedra of type $\mathbf{P}^{1}$ is $\leq 2 \times 24=48$. By a similar argument, the number of different equivalence classes of tetrahedra of type $\mathbf{P}^{2}$ is $\leq 2 \times 48=96$. Hence, the total number of classes of similar tetrahedra in all the $\mathscr{T}^{n}, n \geq 0$, is finite and bounded above by 168 .

From Theorem 4, it follows that $\eta\left(\mathbf{T}_{i}^{n}\right) \geq c_{1} \eta(\mathbf{T})$ for some constant $c_{1}$ that may depend on $\mathbf{T}$. The following theorem establishes that $c_{1}$ is independent of $\mathbf{T}$.

Theorem 5. For any tetrahedron $\mathbf{T}_{i}^{n}$ in $\mathscr{T}^{n}$, we have

$$
\eta\left(\mathbf{T}_{i}^{n}\right) \geq c_{1} \eta(\mathbf{T}),
$$

where $c_{1}$ is a positive constant independent of $\mathbf{T}$.

Proof. Let $M(\mathbf{P}, \mathbf{T})$ and $M(\mathbf{R}, \mathbf{P})$ be the two matrices involved in the affine transformations from $\mathbf{P}$ to $\mathbf{T}$ and $\mathbf{R}$ to $\mathbf{P}$, respectively. Using the notation of Definition 1, we have

$$
T=M(\mathbf{P}, \mathbf{T}) P=M(\mathbf{P}, \mathbf{T}) M(\mathbf{R}, \mathbf{P}) R .
$$

From step (c) of the bisection procedure, the tetrahedron $\mathbf{T}_{i}^{n}$ is transformed from $\mathbf{P}_{i}^{n}$ using $M(\mathbf{P}, \mathbf{T})$, so

$$
T_{i}^{n}=M(\mathbf{P}, \mathbf{T}) P_{i}^{n} .
$$

If $\mathbf{P}_{i}^{n}$ is a tetrahedron of type $\mathbf{P}^{0}$, then with a suitable ordering of vertices of $\mathbf{P}_{i}^{n}$, we have $P_{i}^{n}=\alpha Q P$, where $\alpha$ is a positive constant and $Q$ is an orthogonal matrix, since $\mathbf{P}_{i}^{n}$ is similar to $\mathbf{P}$. If $\mathbf{P}_{i}^{n}$ is a tetrahedron of type $\mathbf{P}^{1}$, then $P_{i}^{n}=\alpha Q M\left(\mathbf{P}, \mathbf{P}_{k}^{1}\right) P$, where $\alpha$ is a positive constant, $Q$ is an orthogonal matrix, and $\mathbf{P}_{k}^{1}$ is any of the two tetrahedra in $\mathscr{P} 1$. If $\mathbf{P}_{i}^{n}$ is a tetrahedron of type $\mathbf{P}^{2}$, then $P_{i}^{n}=\alpha Q M\left(\mathbf{P}, \mathbf{P}_{k}^{2}\right) P$, where $\alpha$ is a positive constant, $Q$ is an orthogonal matrix, and $\mathbf{P}_{k}^{2}$ is any of the four tetrahedra in $\mathscr{P}^{2}$.

Let $\mathbf{R}^{n}$ be the regular tetrahedron of Figure 1 having the same volume as $\mathbf{T}_{i}^{n}$. Then

$$
T_{i}^{n}=M(\mathbf{P}, \mathbf{T}) C M(\mathbf{R}, \mathbf{P}) R^{n},
$$


where $C$ is one of $Q, \sqrt[3]{2} Q M\left(\mathbf{P}, \mathbf{P}_{k}^{1}\right)$, or $\sqrt[3]{4} Q M\left(\mathbf{P}, \mathbf{P}_{k}^{2}\right)$, depending on the type of $\mathbf{P}_{i}^{n}$. By uniqueness, $M(\mathbf{P}, \mathbf{T}) C M(\mathbf{R}, \mathbf{P})=M\left(\mathbf{R}^{n}, \mathbf{T}_{i}^{n}\right)$. From (5) and (6),

$$
\begin{aligned}
\eta(\mathbf{T}) & =\frac{3}{\operatorname{trace}(A(\mathbf{R}, \mathbf{T}))} \\
& =\frac{3}{\operatorname{trace}\left((M(\mathbf{P}, \mathbf{T}) M(\mathbf{R}, \mathbf{P}))^{T} M(\mathbf{P}, \mathbf{T}) M(\mathbf{R}, \mathbf{P})\right)}, \\
\eta\left(\mathbf{T}_{i}^{n}\right) & =\frac{3}{\operatorname{trace}\left(A\left(\mathbf{R}^{n}, \mathbf{T}_{i}^{n}\right)\right)} \\
& =\frac{3}{\operatorname{trace}\left((M(\mathbf{P}, \mathbf{T}) C M(\mathbf{R}, \mathbf{P}))^{T} M(\mathbf{P}, \mathbf{T}) C M(\mathbf{R}, \mathbf{P})\right)} .
\end{aligned}
$$

Let $\|B\|_{F}$ denote the Frobenius norm of matrix $B$ [2], i.e.,

$$
\|B\|_{F}=\left(\operatorname{trace}\left(B^{T} B\right)\right)^{1 / 2}
$$

is the square root of the sum of squares of the elements of $B$. From (8) and (9),

$$
\frac{\eta\left(\mathbf{T}_{i}^{n}\right)}{\eta(\mathbf{T})}=\frac{\|M(\mathbf{P}, \mathbf{T}) M(\mathbf{R}, \mathbf{P})\|_{F}^{2}}{\|M(\mathbf{P}, \mathbf{T}) C M(\mathbf{R}, \mathbf{P})\|_{F}^{2}} .
$$

Since $\|Q\|_{F}^{2}=3$ for a $3 \times 3$ orthogonal matrix $Q$ and $\|A B\|_{F} \leq\|A\|_{F}\|B\|_{F}$ for any $3 \times 3$ matrices $A$ and $B$, we have

$$
\begin{gathered}
\|M(\mathbf{P}, \mathbf{T}) C M(\mathbf{R}, \mathbf{P})\|_{F}^{2} \leq\|M(\mathbf{P}, \mathbf{T}) M(\mathbf{R}, \mathbf{P})\|_{F}^{2}\left\|M^{-1}(\mathbf{R}, \mathbf{P}) C M(\mathbf{R}, \mathbf{P})\right\|_{F}^{2} \\
\leq 3\|M(\mathbf{P}, \mathbf{T}) M(\mathbf{R}, \mathbf{P})\|_{F}^{2}\left\|M^{-1}(\mathbf{R}, \mathbf{P})\right\|_{F}^{2}\|M(\mathbf{R}, \mathbf{P})\|_{F}^{2} \max \left(1, s_{1}, s_{2}\right),
\end{gathered}
$$

where

$$
s_{1}=2^{2 / 3} \max _{1 \leq k \leq 2}\left(\left\|M\left(\mathbf{P}, \mathbf{P}_{k}^{1}\right)\right\|_{F}^{2}\right) \quad \text { and } \quad s_{2}=2^{4 / 3} \max _{1 \leq k \leq 4}\left(\left\|M\left(\mathbf{P}, \mathbf{P}_{k}^{2}\right)\right\|_{F}^{2}\right) .
$$

From (10) and (11), we get $\eta\left(\mathbf{T}_{i}^{n}\right) / \eta(\mathbf{T}) \geq c_{1}$, where

$$
c_{1}=\frac{1}{\left[3\left\|M^{-1}(\mathbf{R}, \mathbf{P})\right\|_{F}^{2}\|M(\mathbf{R}, \mathbf{P})\|_{F}^{2} \max \left(1, s_{1}, s_{2}\right)\right]} .
$$

Theorem 6. For any tetrahedron $\mathrm{T}_{i}^{n}$ in $\mathscr{T}^{n}$, we have

$$
\delta\left(\mathbf{T}_{i}^{n}\right) \leq c_{2}(1 / 2)^{n / 3} \delta(\mathbf{T}),
$$

where $c_{2}$ is a positive constant independent of $\mathbf{T}$. 
Proof. By Theorem 1,

$$
\eta(\mathbf{T})=\frac{12(3 v)^{2 / 3}}{\sum_{i=1}^{6} l_{i, 0}^{2}}
$$

where $v$ is the volume of $\mathbf{T}$ and the $l_{i, 0}$ are the lengths of the edges of $\mathbf{T}$. At each level of bisection, the volume of a subtetrahedron is decreased by a factor of 2 , so after $n$ levels of bisection,

$$
\eta\left(\mathbf{T}_{i}^{n}\right)=\frac{12(1 / 2)^{2 n / 3}(3 v)^{2 / 3}}{\sum_{i=1}^{6} l_{i, n}^{2}},
$$

where the $l_{i, n}$ are the lengths of the edges of $\mathbf{T}_{i}^{n}$. Substituting (12) and (13) in (7) yields

$$
\sum_{i=1}^{6} l_{i, n}^{2} \leq\left(1 / c_{1}\right)(1 / 2)^{2 n / 3} \sum_{i=1}^{6} l_{i, 0}^{2}
$$

where $c_{1}$ is a positive constant independent of $\mathbf{T}$. So

$$
\delta\left(\mathbf{T}_{i}^{n}\right)^{2} \leq \sum_{i=1}^{6} l_{i, n}^{2} \leq\left(1 / c_{1}\right)(1 / 2)^{2 n / 3} \sum_{i=1}^{6} l_{i, 0}^{2} \leq\left(1 / c_{1}\right)(1 / 2)^{2 n / 3} 6 \delta(\mathbf{T})^{2} .
$$

That is, $\delta\left(\mathbf{T}_{i}^{n}\right) \leq c_{2}(1 / 2)^{n / 3} \delta(\mathbf{T})$, where $c_{2}=\sqrt{6 / c_{1}}$.

\section{EsTIMATE OF CONSTANTS}

In this section, we obtain an estimate of the constant $c_{1}$ in Theorem 5 , which then provides an estimate of the constant $c_{2}$ in Theorem 6. Our derivation of the estimate of $c_{1}$ starts from (10) in the proof of Theorem 5, since (11) provides an estimate that is too small. At the first two levels of bisection, we use the tetrahedra $\mathbf{P}_{1}^{1}\left(p_{12}, p_{1}, p_{3}, p_{0}\right)$ and $\mathbf{P}_{1}^{2}\left(p_{12}, p_{0}, p_{1}, p_{13}\right)$.

By the singular value decomposition [2],

$$
M(\mathbf{P}, \mathbf{T})=Q_{1} \operatorname{diag}\left(\sqrt{\lambda_{1}}, \sqrt{\lambda_{2}}, \sqrt{\lambda_{3}}\right) Q_{2},
$$

where $Q_{1}, Q_{2}$ are orthogonal matrices and $\lambda_{1}, \lambda_{2}, \lambda_{3}$ are the eigenvalues of $A(\mathbf{P}, \mathbf{T})\left(\sqrt{\lambda_{1}}, \sqrt{\lambda_{2}}, \sqrt{\lambda_{3}}\right.$ are the singular values of $\left.M(\mathbf{P}, \mathbf{T})\right)$. Similarly,

$$
\begin{array}{r}
M(\mathbf{R}, \mathbf{P})=Q_{3} \operatorname{diag}\left(\sqrt{\mu_{11}}, \sqrt{\mu_{12}}, \sqrt{\mu_{13}}\right) Q_{4}, \\
\sqrt[3]{2} M\left(\mathbf{P}, \mathbf{P}_{1}^{1}\right) M(\mathbf{R}, \mathbf{P})=Q_{5} \operatorname{diag}\left(\sqrt{\mu_{21}}, \sqrt{\mu_{22}}, \sqrt{\mu_{23}}\right) Q_{6},
\end{array}
$$

and

$$
\sqrt[3]{4} M\left(\mathbf{P}, \mathbf{P}_{1}^{2}\right) M(\mathbf{R}, \mathbf{P})=Q_{7} \operatorname{diag}\left(\sqrt{\mu_{31}}, \sqrt{\mu_{32}}, \sqrt{\mu_{33}}\right) Q_{8},
$$

where the $Q_{i}$ are orthogonal matrices and the $\mu_{i j}$ are eigenvalues. It follows that

$$
\begin{aligned}
& \|M(\mathbf{P}, \mathbf{T}) M(\mathbf{R}, \mathbf{P})\|_{F}^{2} \\
& \quad=\left\|\operatorname{diag}\left(\sqrt{\lambda_{1}}, \sqrt{\lambda_{2}}, \sqrt{\lambda_{3}}\right) Q_{2} Q_{3} \operatorname{diag}\left(\sqrt{\mu_{11}}, \sqrt{\mu_{12}}, \sqrt{\mu_{13}}\right)\right\|_{F}^{2},
\end{aligned}
$$




$$
\begin{aligned}
& \|M(\mathbf{P}, \mathbf{T}) C M(\mathbf{R}, \mathbf{P})\|_{F}^{2} \\
& \quad=\left\|\operatorname{diag}\left(\sqrt{\lambda_{1}}, \sqrt{\lambda_{2}}, \sqrt{\lambda_{3}}\right) Q_{9} \operatorname{diag}\left(\sqrt{\mu_{i 1}}, \sqrt{\mu_{i 2}}, \sqrt{\mu_{i 3}}\right)\right\|_{F}^{2},
\end{aligned}
$$

where $Q_{9}$ is an orthogonal matrix and $i$ is 1,2 , or 3. For any orthogonal matrix $Q$, let $S=\operatorname{diag}\left(\sqrt{\lambda_{1}}, \sqrt{\lambda_{2}}, \sqrt{\lambda_{3}}\right) Q \operatorname{diag}\left(\sqrt{\mu_{i 1}}, \sqrt{\mu_{i 2}}, \sqrt{\mu_{i 3}}\right)$. By carrying out the two matrix multiplications, it is easy to see that

$$
\begin{aligned}
& \min \left(\mu_{i 1}, \mu_{i 2}, \mu_{i 3}\right)\left(\lambda_{1}+\lambda_{2}+\lambda_{3}\right) \\
& \quad \leq\|S\|_{F}^{2} \leq \max \left(\mu_{i 1}, \mu_{i 2}, \mu_{i 3}\right)\left(\lambda_{1}+\lambda_{2}+\lambda_{3}\right) .
\end{aligned}
$$

From (14), (15), and (16), it follows that

$$
\begin{gathered}
\|M(\mathbf{P}, \mathbf{T}) M(\mathbf{R}, \mathbf{P})\|_{F}^{2} \geq\left(\lambda_{1}+\lambda_{2}+\lambda_{3}\right) \min _{1 \leq j \leq 3}\left(\mu_{1 j}\right), \\
\|M(\mathbf{P}, \mathbf{T}) C M(\mathbf{R}, \mathbf{P})\|_{F}^{2} \leq\left(\lambda_{1}+\lambda_{2}+\lambda_{3}\right) \max _{1 \leq i, j \leq 3}\left(\mu_{i j}\right) .
\end{gathered}
$$

Substituting (17) and (18) into (10), yields

$$
\frac{\eta\left(\mathbf{T}_{i}^{n}\right)}{\eta(\mathbf{T})} \geq \frac{\min _{1 \leq j \leq 3}\left(\mu_{1 j}\right)}{\max _{1 \leq i, j \leq 3}\left(\mu_{i j}\right)}
$$

We now compute the eigenvalues $\mu_{i j}$. Using the coordinates of Figures 1 and 3 , we obtain

$$
M(\mathbf{R}, \mathbf{P})=P R^{-1}=\frac{1}{\sqrt[3]{2}}\left[\begin{array}{ccc}
0 & 2 & 0 \\
-\sqrt{6} / 3 & 0 & -\sqrt{3} / 6 \\
0 & 0 & \sqrt{6} / 2
\end{array}\right] .
$$

So in decreasing order, $\mu_{11}=4 / \sqrt[3]{4}, \mu_{12}=(9+\sqrt{17}) /(8 \sqrt[3]{4})$, and $\mu_{13}=$ $(9-\sqrt{17}) /(8 \sqrt[3]{4})$. From

$$
\sqrt[3]{2} M\left(\mathbf{P}, \mathbf{P}_{1}^{1}\right) M(\mathbf{R}, \mathbf{P})=\left[\begin{array}{ccc}
-\sqrt{3} / 3 & 1 & -\sqrt{6} / 6 \\
0 & 0 & \sqrt{3} / 2 \\
\sqrt{3} / 3 & 1 & -\sqrt{6} / 6
\end{array}\right]
$$

we get $\mu_{21}=2, \mu_{22}=(7+\sqrt{17}) / 8$, and $\mu_{23}=(7-\sqrt{17}) / 8$ in decreasing order. From

$$
\sqrt[3]{4} M\left(\mathbf{P}, \mathbf{P}_{1}^{2}\right) M(\mathbf{R}, \mathbf{P})=\sqrt[3]{2}\left[\begin{array}{ccc}
-\sqrt{3} / 3 & -1 & -\sqrt{6} / 12 \\
\sqrt{6} / 6 & -\sqrt{2} / 2 & -\sqrt{3} / 6 \\
0 & 0 & \sqrt{6} / 4
\end{array}\right]
$$

we get $\mu_{31}=\sqrt[3]{4}(2+\sqrt{2}) / 2, \quad \mu_{32}=\sqrt[3]{4} / 2$, and $\mu_{33}=\sqrt[3]{4}(2-\sqrt{2}) / 2$ in decreasing order.

From (19), $\eta\left(\mathbf{T}_{i}^{n}\right) / \eta(\mathbf{T}) \geq c_{1}=\mu_{13} / \mu_{31}=\sqrt[3]{4}(9-\sqrt{17})(2-\sqrt{2}) / 32=$ 0.1417. Then it follows from $c_{2}=\sqrt{6 / c_{1}}$ that $c_{2}=6.5068$. By using a different approach, it may be possible to obtain better estimates of $c_{1}$ and $c_{2}$, but we believe that our current estimates can be improved by at most a small factor (unless a better $\mathbf{P}$ tetrahedron can be found). 


\section{ACKNOWLEDGMENT}

The authors would like to thank Wenping Wang for many helpful discussions.

\section{BIBLIOGRAPHY}

1. A. Adler, On the bisection method for triangles, Math. Comp. 40 (1983), 571-574.

2. G. H. Golub and C. F. Van Loan, Matrix computations, 2nd ed., Johns Hopkins University Press, Baltimore, MD, 1989.

3. B. Joe, Delaunay versus max-min solid angle triangulations for three-dimensional mesh generation, Internat. J. Numer. Methods Engrg. 31 (1991), 987-997.

4. _ Three-dimensional boundary-constrained triangulations, Artificial Intelligence, Expert Systems, and Symbolic Computing (E. N. Houstis and J. R. Rice, eds.), Elsevier Science Publishers, 1992, pp. 215-222.

5. B. Kearfott, $A$ proof of convergence and an error bound for the method of bisection in $\mathbf{R}^{n}$, Math. Comp. 32 (1978), 1147-1153.

6. M.-C. Rivara, Mesh refinement processes based on the generalized bisection of simplices, SIAM J. Numer. Anal. 21 (1984), 604-613.

7. __ Algorithms for refining triangular grids suitable for adaptive and multigrid techniques, Internat. J. Numer. Methods Engrg. 20 (1984), 745-756.

8. _ A grid generator based on 4-triangles conforming mesh-refinement algorithms, Internat. J. Numer. Methods Engrg. 24 (1987), 1343-1354.

9. I. G. Rosenberg and F. Stenger, $A$ lower bound on the angles of triangles constructed by bisecting the longest side, Math. Comp. 29 (1975), 390-395.

10. M. Senechal, Which tetrahedra fill space?, Math. Mag. 54 (1981), 227-243.

11. M. Stynes, On faster convergence of the bisection method for all triangles, Math. Comp. 35 (1980), 1195-1201.

Department of Computing Science, University of Alberta, Edmonton, Alberta, Canada T6G 2H 1

E-mail address: anwei@cs.ualberta.ca

E-mail address: barryecs.ualberta.ca 